\title{
Spontaneous temporomandibular joint herniation into the external auditory canal through a persistent foramen tympanicum (huschke): otologic features
}

\begin{abstract}
A persistent foramen tympanicum (Huschke) is an anatomic variation located in the anterior inferior portion of the bony external ear canal.

We present a case of symptomatic temporomandibular joint (TMJ) herniation into the right external auditory canal though an enlarged osseous defect. The herniated retrodiscal temporo-mandibular joint (TMJ) tissue moved backward when the patient's mouth was closed, and forward, when opened. Imaging findings were useful for differentiating TMJ herniation from salivary fistula or a true granuloma of the anterior bony canal wall near the tympanic membrane.

The purpose of this presentation is to alert otologists that a large granuloma near the anterior inferior or anterior superior portion of the bony ear canal adjacent to the anterior superior tympanic membrane can be a herniation of either the parotid gland or in this case, the temporomandibular joint capsule. Several attempts at removal of this through the ear canal failed because of the initially incorrect diagnosis of a primary lesion of the ear canal. Only after considerable thought was it realized that this was a rare herniation of the temporomandibular joint capsule through a patent foramen of Huschke.
\end{abstract}

Keywords: foramen of huschke, otorrhea, joint capsule
Volume 2 Issue 4 - 2015

\author{
Joel M Bernstein,' Thom Loree, ${ }^{2}$ Gregory \\ Tymchak $^{3}$ \\ 'Departments of Otolaryngology and Pediatrics, State \\ University of New York, USA \\ ${ }^{2}$ Department of Otolaryngology, State University of New York, \\ USA \\ ${ }^{3}$ Department of Radiology, State University of New York, USA
}

Correspondence: Joel M Bernstein, Departments of

Otolaryngology and Pediatrics, State University of New York, 2430 North Forest Road, Getzville, NYI 4068, New York, USA, Tel (7I6) 636-76I3, Fax (7I6) 636-76I6,

Email jbernste@acsu.buffalo.edu

Received: April 0I, 20I5 | Published: May 08, 2015

\section{Introduction}

Emil Huschke (December 14, 1797-June 19, 1858) was a German anatomist and embryologist who was a native of Weimar. ${ }^{1} \mathrm{He}$ was the first to describe a handful of anatomical structures that now contain his name, including Huschke's foramen: an opening in the floor of the bony part of the external acoustic meatus in the vicinity of the tympanic membrane. Although it is normally closed in adults, at least $5 \%$ of children have a patent foramen of Huschke. ${ }^{2}$

\section{Case report}

A 58 year-old woman, presented for otolaryngologic evaluation with symptoms of right ear canal blockage, slight hearing loss and serous otorrhea. She reported that 6months earlier, while eating a peanut, she experienced a sudden cracking sensation in the right ear. This was followed by fullness and pressure in the right ear canal which was accompanied by minimal drainage from the canal. Her past medical history was remarkable for a history of mild but noticeable asymmetry since childhood.

Examination revealed granular mass in the right ear canal in the anterior superior obstruction about $50 \%$ of the tympanic membrane.

She was placed on an antibiotic ear drop regimen and a CT scan of the temporal bones was obtained. The reading of the CT scan identified a mass in the external ear canal but failed to identify any defect in the bony canal wall adjacent to the tympanic membrane.

The patient was referred for further evaluation. At examination by the first author, the lesion was circular, red and wet, measuring $7-8 \mathrm{~mm}$. in diameter. It occluded the anterior half of the right tympanic membrane and was draining serous fluid. There was no purulence. Gentle palpation of the lesion demonstrated that it was not attached to the tympanic membrane. The lesion was originally diagnosed as a granuloma of the right bony ear canal which was adjacent to the tympanic membrane in the anterior superior portion of the bony ear canal.

With the diagnosis of granuloma, the patient was scheduled for excision of the lesion under general anesthesia. This was performed two weeks following presentation. Photograph of the lesion is seen in Figure 1. It demonstrates a small blood clot on the superior aspect of the tympanic membrane. The inferior portion of the tympanic membrane is normal and there is a cystic-like lesion in the anterior superior canal wall. Interestingly, the inferior tympanic membrane is normal. At this time, it was felt that the lesion was primarily a granuloma arising from the ear canal.

Shortly after surgery, the packing dressing was removed. The right ear canal was almost healed. The patient's hearing returned to normal. There was no difference in hearing between the left and right ears. However, one month later, a recurrence of the granuloma of the anterior canal wall adjacent to the tympanic membrane was again visualized. At this time, because there was a thin, clear serous discharge, it was thought that the lesion could represent a herniation of the parotid gland. The pathologist was alerted to determine whether or not any evidence of parotid gland was seen in the histopathology.

A second operation was performed. This time a $2-2.5 \mathrm{~mm}$. granuloma was present in the anterior superior portion of the bony ear canal adjacent to the tympanic membrane. Although the anterior half of the tympanic membrane was swollen and injected, the lesion was not attached to the tympanic membrane.

The possibility of a herniation of the tissue through a patent foramen of Huschke was not considered at this time. The pathology report did not demonstrate any parotid tissue. An audiogram revealed 
normal hearing in both ears. The discrimination scores were $100 \%$ bilaterally.

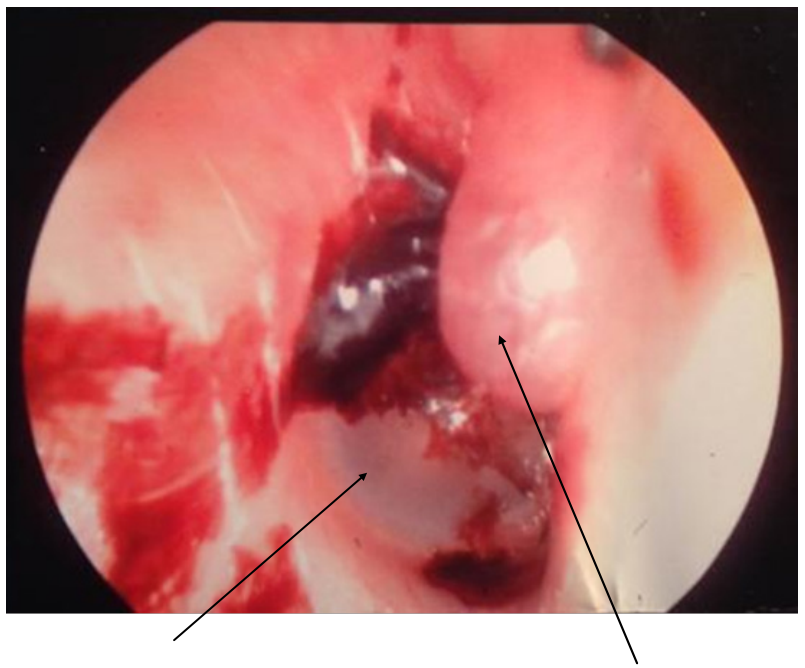

Figure I Photograph of right external ear canal of patient demonstrating a herniation of the right TMJ capsule into the anterior canal wall (right arrow) through a patent foramen of Huschke. The lower half of the tympanic membrane is normal (left arrow). The superior portion of the tympanic membrane is covered with a blood clot

Two months later, a third operative procedure was performed for excision of a further recurrence of the granuloma in the anterior superior portion of the ear canal. Following this third procedure, the idea of a herniation through a bony defect in the anterior canal wall was finally considered. A second CAT scan of the temporal bones was performed. This scan revealed a soft tissue density closely associated with the tympanic membrane in the external auditory canal on the right just anterior to it and what appeared to be a small defect or thinning of the anterior canal wall just posterior to the temporomandibular joint. Axial and coronal views of the defect in the anterior wall are shown in Figures 2 (A \& B) and demonstrate conclusively the presence of a patent foramen of Huschke. Further examination revealed motion of the lesion with mastication. The patient's facial asymmetry was determined to be a consequence of mandibular asymmetry.

Finally, the correct diagnosis was made. Although the appearance of the lesion was that of a granuloma arising from the bony ear canal, the correct diagnosis was a herniation of the capsule of the temporomandibular joint through a patent foramen of Huschke which had become inflamed and contaminated by commensal bacteria in the ear canal.

With this diagnosis, further surgery was performed. The right temporo-mandibular joint was exposed via a pre-auricular approach. The right temporo-mandibular joint capsule disruption with a patent foramen of Huschke was identified. The right temporo-mandibular joint was reconstructed with a right conchal cartilage graft.

A conchal graft was elevated and harvested and the anterior skin of the tragus and subcutaneous tissue was elevated and a $3 \times 2 \mathrm{~cm}$ conchal cartilage graft was placed between the joint and the bony external canal. The post-operative course was uneventful with the exception of the patient's altered sensation during mastication. No significant change in dental occlusion was present. Two months after surgery examination no longer demonstrated movement of the canal wall with mastication. The right ear canal was clean, dry and entirely normal.
One year following the repair of the patent foramen of Huschke with conchal cartilage, the lesion and associated symptoms have not recurred.
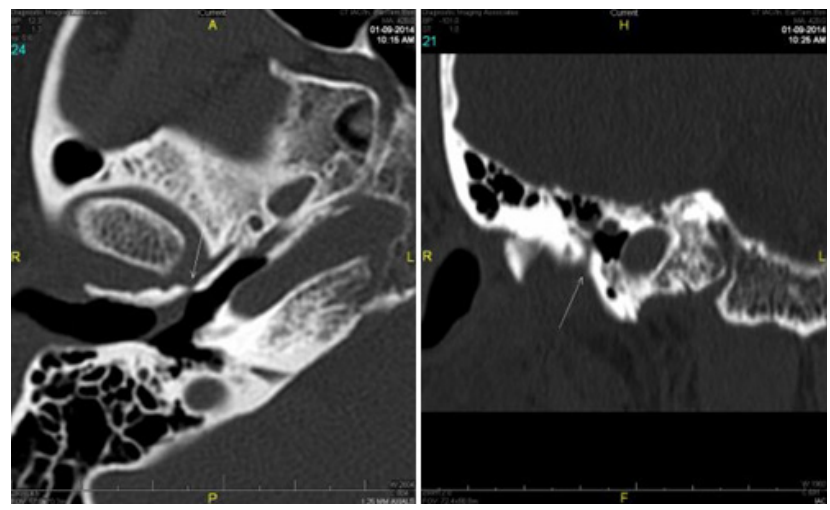

Figure 2a Axial image at the level of the condylar head of the mandible at the Tmj joint. The arrow depicts a small defect in the anterior wall of the external auditory canal with soft tissue density that is contigious with the Tmj joint. This is closely approximated to the soft tissue density that abuts the tympanic membrane.

Figure 2b Reconstructed para-coronal images through the anterior wall of the external auditory canal just posterior to the condylar head of the mandible at the level of the Tmj Joint. Arrow depicts a patent foramen of Huschke.

In retrospect, the patient had a defect in the anterior superior bony canal wall consistent with a patent foramen of Huschke resulting in herniation of the TMJ capsule, most likely a consequence of chronic trauma to the temporo-mandibular joint secondary to congenital asymmetry and subsequent injury to the TMJ capsule earlier in life. ${ }^{3}$

\section{Discussion}

Persistent foramen tympanicum, also known as foramen of Huschke is a developmental osseous defect or dehiscence located in the anterior canal wall. ${ }^{3}$ Thirty one cases have been described in the literature of radiology, otolaryngology and dentistry over the last 40 years. Of the cases described, it is interesting that the defect is usually considered to be in the anterior inferior portion of the bony canal wall, very close to the tympanic membrane. However, in our case, this defect was not in the anterior inferior portion of the ear canal, but in the anterior superior portion of the ear canal.

The incidence of foramen of Huschke is approximately $7.2 \%$ in cadaveric studies and approximately $4.6 \%$ in high resolution temporal bone CT scans. ${ }^{4}$ The tympanic bone forms an incomplete u-shape at birth. Anterior and posterior prominences of the $\mathrm{u}$-shaped bone grow and fuse at the age of about 1 year and the external auditory canal finally closes by the age of 5 . However, persistent osseous dehiscence is occasionally observed after the age of 5 years. ${ }^{5}$

The foramen of Huschke is a route of communication between the external ear canal and the infratemporal fossa, and it may also provide a route for tumors and infection to spread. ${ }^{6}$ Herniation of a first branchial arch cyst migrating through this defect has also been reported. ${ }^{7}$ Another abnormal condition which is the one presented in this communication is a spontaneous temporomandibular joint herniation into the external ear canal through the osseous dehiscence. ${ }^{8}$ This is, in most cases, a very rare condition that is observed in only $0.4 \%$ of the population. Approximately 31 cases have been reported in the medical literature. ${ }^{9}$ 
One hypothesis suggests that initially, foramen of Huschke is probably too small for herniation but mastication movements increase the size of the defect resulting in soft tissue herniation. In addition, ectopic salivary gland tissue in the anterior inferior external auditory canal may promote the development salivary fistulas between the parotid gland and the external auditory canal. ${ }^{10}$ Otorrhea is usually associated with temporomandibular joint movement during mastication and is not specific to either salivary or temporomandibular joint fistulas.

Evaluation of the external auditory canal while the jaw is moving may help in the diagnosis of foramen of Huschke. The movement of the herniation is characteristic and can be easily identified clinically. Whereas the herniation usually protrudes into the external auditory canal forming a dome-shaped polyp during closing of the mouth, it is retracted because of the negative pressure of the retrodiscal space during anterior translation of the temporomandibular joint, similar to other anatomic c communications.

The major purpose of this communication is to alert otolaryngologists that a large red mass in the anterior superior or anterior inferior canal wall may be the result of herniation of either the parotid gland or in this case, the temporomandibular joint capsule and can be easily misdiagnosed as a lesion arising from the ear canal, as was the present case.

\section{Summary}

A 58year-old woman presented with a granuloma or granulation tissue of the anterior superior canal wall which was initially diagnosed incorrectly by as a mass arising from the ear canal itself. Only after failure of removal of this granulomatous lesion on three occasions was the otologist alerted to the idea that a defect in the anterior canal wall at the foramen tympanicum (or foramen of Huschke) was considered. The correct diagnosis was then documented by CT scan of the temporal bones. Following the identification of a patent foramen of Huschke, the patient was successfully treated with closure of the defect.

The purpose of this communication is not simply to present another case of a patent foramen of Huschke, but to alert otolaryngologists to this moderately rare condition. If a granuloma is suspected in the position of the anterior inferior or anterior superior bony canal wall and free of the tympanic membrane, a patent foramen of Huschke should be thought of and proper radiographic confirmation be undertaken.

\section{Acknowledgments}

None.

\section{Conflicts of interest}

The authors declare that there are no conflicts of interest.

\section{Funding details}

None.

\section{References}

1. Wikipedia Foundation, Inc., 2013.

2. Lacuot A, Marsot-Dupuch K, Smoker WR, et al. Foramen Tympanicum, or foramen of Huschke: pathologic cases in anatomic CT study. ANJR Am J Neuroradiol. 2005;26(6):1317-1323.

3. Akcam T, Hidir Y, Ilica AT, et al. Temporomandibular joint herniation into the external ear canal through foramen of Huschke. Auris Nasus Larynx. 2011;38(5):646-649.

4. Nakasato T, Nakayama T, Kikuchi $\mathrm{K}$, et al. Spontaneous temporomandibular joint herniation into the external canal through a persistent foramen tympanicum (Huschke): radiographic features. $J$ Comput Assist Tomogr. 2013;37(1):111-113.

5. Humphrey LT, Scheuer L. Age of closure of the foramen of Huschke: an osteological study. Int J Osteoarchaeology. 2006;16(1):47-60.

6. Psillas G, Guyot JP. Spontaneous temporomandibular joint herniation into the external ear canal. Ann Otolaryngol Chir Cervicofac. 2007;124(6):305-308.

7. Silva RC, Collins WO. Persistent foramen of Huschke mimicking a Branchial Cleft Anomaly. Arch Otolaryngol Head Neck Surg. 2012;138(12):1176-1179.

8. Kim TH, Lee SK, Kim SJ, et al. A case of spontenous temporomandibular joint herniation into the external auditory canal with clicking sound. Korean J Audiol. 2013;17(2):90-93.

9. Srimani P, Mukherjee P, Ghosh E, et al. Variant presentations of foramen of Huschke in 7 adult human crania. Int J Anatomic Variations. 2013;6:120-123.

10. DeZoysa N, Vasani S, Kaniyur S, et al. Gustatory otorrhea: a rare case of congenital external ear salivary fistula. J Laryngol Otol. 2009;123(12):1371-1374. 